\title{
Convergence of Iterative of Split-Operator Approaches for Approximating Nonlinear Reactive Transport Problems
}

\author{
Joseph F. Kanney, Cass T. Miller ${ }^{1}$ \\ Center for the Advanced Study of the Environment, Department of Environmental \\ Sciences and Engineering, University of North Carolina, Chapel Hill, North \\ Carolina 27599-7400, USA \\ C. T. Kelley \\ Center for Research in Scientific Computation, Department of Mathematics, \\ North Carolina State University, Raleigh, NC, 27695-8205
}

\begin{abstract}
Numerical solutions to nonlinear reactive solute transport problems are often computed using split-operator ( $\mathrm{SO}$ ) approaches, which separate the transport and reaction processes. This uncoupling introduces an additional source of numerical error, known as the splitting error. The iterative split-operator (ISO) algorithm removes the splitting error through iteration. Although the ISO algorithm is often used, there has been very little analysis of its convergence behavior. This work uses theoretical analysis and numerical experiments to investigate the convergence rate of the iterative split-operator approach for solving nonlinear reactive transport problems.
\end{abstract}

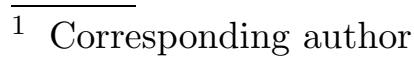

2 E-mail addresses: joe_kanney@unc.edu (J. F. Kanney), Tim_Kelley@ncsu.edu (C.

T. Kelley), casey_miller@unc.edu(C. T. Miller), 


\section{Notation}

\section{Roman Letters}

$C \quad$ volume averaged aqueous phase solute concentration $\left[M L^{3}\right]$

$D_{x} \quad$ dispersion coefficient in $x$ direction $\left[L^{2} T{ }^{1}\right]$

$e \quad$ difference of two general functions [ 1$]$

$E \quad$ error in a numerical solution [ 1]

$F \quad$ general operator acting on $\mathbb{R}^{n}$-valued functions [ ]

$f \quad$ forcing function in simple ODE [ ]

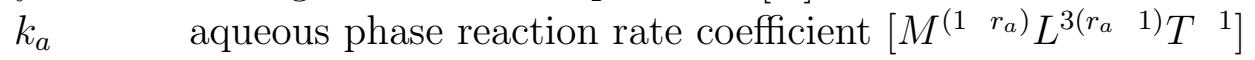

$k_{f} \quad$ rapidly sorbing solid phase fraction reaction rate coefficient $\left[\begin{array}{ll}T^{1}\end{array}\right]$

$k_{s} \quad$ slowly sorbing solid phase fraction reaction rate coefficient $\left[\begin{array}{ll}T & 1\end{array}\right]$

$K \quad$ general constant in algorithm analysis [ ]

$K_{f} \quad$ rapidly sorbing solid phase fraction Freundlich coefficient $\left[L^{3 n_{f}} M n_{f}\right]$

$K_{s} \quad$ slowly sorbing solid phase fraction Freundlich coefficient $\left[\begin{array}{lll}L^{3 n_{s}} M & n_{s}\end{array}\right]$

$L_{t} \quad$ transport operator [ ]

$L_{r 1} \quad$ reaction operator [ ]

$L_{r 2} \quad$ reaction operator [ ]

$M \quad$ general constant in algorithm analysis [ ]

$n_{e} \quad$ number of equations [ ]

$n_{f} \quad$ rapidly sorbing solid phase fraction Freundlich exponent [ ]

$n_{s} \quad$ slowly sorbing solid phase fraction Freundlich exponent [ ]

O asymptotic order [ ]

$Q \quad$ rate of convergence of ISO iterations [ ]

$r_{a} \quad$ aqueous phase reaction order [ ]

$r_{f} \quad$ rapidly sorbing solid phase fraction reaction order [ ]

$r_{s} \quad$ slowly sorbing solid phase fraction reaction order [ ]

$R \quad$ general operator acting on $\mathbb{R}^{n}$-valued functions [ ]

$R_{f} \quad$ retardation factor [ ]

$\mathbb{R}^{n} \quad$ n-dimensional Euclidean space $\left[L^{n}\right]$

$t \quad$ temporal coordinate $[T]$

$t_{s} \quad$ simulation length $[T]$

$\Delta t \quad$ time increment $[T]$

$u \quad$ general $\mathbb{R}^{n}$-valued function [ ]

$v \quad$ dependent variable in simple ODE [ ]

$v_{x} \quad$ pore velocity component in $x$ direction in $\mathrm{PDE}\left[L T T^{1}\right]$

$w \quad$ dependent variable in simple ODE [ ]

$x \quad$ spatial coordinate $[L]$

$x_{l} \quad$ one-dimensional spatial domain length $[L]$

$\Delta x \quad$ space increment $[L]$ 


\section{Greek Letters}

$\alpha \quad$ first order mass transfer coefficient for mass transfer between aqueous phase and slowly sorbing solid phase fraction $\left[\begin{array}{ll}T & 1\end{array}\right]$

$\|\epsilon\|_{k} \quad$ discrete vector norm of index $\mathrm{k}$, where $k=1,2, \infty$ [ ]

$\omega \quad$ mass averaged solid phase solute concentration $\left[M M^{1}\right]$

$\gamma \quad$ Lipschitz constant [ ]

$\rho \quad$ solid phase particle density $\left[M L^{3}\right]$

$\Phi \quad$ denotes the solution $\left(C, \omega_{s}\right)$ of the system of transport equations [ ]

$\rho_{b} \quad$ solid phase bulk density $\left[M L^{3}\right]$

$\tau \quad$ temporal discretization error of numerical solution [ ]

$\theta \quad$ solid phase porosity (void space volume fraction) [ ]

\section{Subscripts}

a qualifier denoting quantity in aqueous phase

$f \quad$ qualifier denoting quantity in rapidly sorbing solid phase fraction

$i \quad$ iteration index in ISO algorithm

$r 1 \quad$ operator qualifier denoting reaction

$r 2 \quad$ operator qualifier denoting reaction

$s \quad$ qualifier denoting quantity in slowly sorbing solid phase fraction

$t \quad$ operator qualifier denoting transport

0 qualifier denoting value of quantity at $x=0$, the inlet of onedimensional domain

Superscripts

* qualifier denoting an exact solution

$0 \quad$ qualifier denoting quantity evaluated at $t=0$

$1 / 2$ qualifier denoting the quantity evaluated after the transport solve in ISO algorithm

$+\quad$ qualifier denoting the quantity evaluated after the reaction solve in ISO algorithm

$c \quad$ qualifier denoting the quantity evaluated using the current approximate solution

e qualifier denoting quantity measured at thermodynamic equilibrium

qualifier denoting quantity obtained using intermediate values of dependent variables in split-operator algorithms

\section{Abbreviations}

1D one-dimensional 


$\begin{array}{ll}\text { 2D } & \text { two-dimensional } \\ \text { 3D } & \text { three-dimensional } \\ \text { AE } & \text { algebraic equation } \\ \text { ADRE } & \text { advection-dispersion-reaction equation } \\ \text { ASO } & \text { alternating split-operator numerical solution algorithm } \\ \text { CD } & \text { central difference discretization of advection term } \\ \text { CN } & \text { Crank-Nicolson temporal discretization } \\ \text { FC } & \text { fully coupled numerical solution algorithm } \\ \text { ISO } & \text { iterative split-operator numerical solution algorithm } \\ \text { NI } & \text { Newton iteration } \\ \text { NRTP } & \text { nonlinear reactive transport problem } \\ \text { ODE } & \text { ordinary differential equation } \\ \text { PDE } & \text { partial differential equation } \\ \text { SIA } & \text { sequential iterative approach } \\ \text { SO } & \text { split-operator numerical solution approach } \\ \text { SNIA } & \text { sequential noniterative approach } \\ \text { SSO } & \text { sequential split-operator numerical solution algorithm }\end{array}$

\section{INTRODUCTION}

Nonlinear reactive transport problems (NRTP's), i.e., problems involving advective and diffusive/dispersive solute transport combined with nonlinear homogeneous and/or heterogeneous reactions, are important in many application areas. Examples include transport of contaminants, dissolved minerals, and microorganisms in groundwater systems $[1,13,15,16,25,40,44,53,60,71]$; contaminant and nutrient transport in oceans, lakes and rivers [18, 19, 31, 41, $57,75]$; pollution transport in the atmosphere $[9,26,33,63,72]$; as well as a variety of industrial applications, e.g. [3, 10, 12, 34, 35, 46, 52]. The governing system of equations for NRTP's typically consist of partial differential equations (PDE's) for transport coupled with algebraic equations (AE's) and/or ordinary differential equations (ODE's) describing chemical equilibrium and chemical kinetics, respectively. These systems seldom admit analytical solutions, so numerical solution techniques are commonly used. Problems that are advective dominated or involve stiff chemical reactions are difficult to solve, even numerically. For large-scale problems and/or problems involving multiple species, the computational expense of obtaining accurate solutions can be substantial [77].

There are two fundamentally different algorithmic approaches to the numerical solution of NRTP's: (1) the fully coupled (FC) approach in which the discrete forms of the governing equations are solved as a single system; and (2) the SO approach, which uses a time-splitting method to uncouple and separately solve the discretized governing equations for transport and reaction. The SO 
algorithm is very attractive for large, multidimensional problems with multiple species because the memory requirements of the individual substeps of the uncoupled problem are significantly smaller than the FC approach [5, 65, 77]. Furthermore, the SO approach allows one to combine a transport-only computer code with a chemistry-only computer code with minimal effort $[8,30]$. The SO approach also lends itself to parallel computation, since the reaction step may be computed independently at each node in the computational grid [77]. Due to these advantages, SO approaches have been widely used to solve NRTP's [36, 65].

A significant drawback of the SO approach is that decoupling the governing equations introduces an additional source of numerical error, referred to as splitting error [70]. The splitting error has been shown to depend upon the order of the solution of the transport and reaction subproblems [5, 70]. Furthermore, the splitting error may be removed by iteration [24], which we refer to as the iterative split-operator (ISO) algorithm. The applicability of ISO depends mainly on the stability requirements and the rate of convergence of the iterations $[24,77]$; others have reported difficulties in getting the iterative procedure to converge [21, 27, 65]. Despite this difficulty, and despite the wide application of SO approaches, there are relatively few studies of the convergence, computational accuracy, and efficiency of the method for general nonlinear problems. There are even fewer studies of the stability and convergence properties of the ISO algorithm.

The overall goal of the work reported here is to investigate the convergence of the ISO algorithm for NRTP's. The specific objectives we pursue are (1) to perform a theoretical analysis of the convergence rate for the ISO algorithm solution of a general NRTP form; and (2) to investigate the implications of the analytical results through numerical experiments using a simplified ODE model and a model NRTP with features relevant to a wide class of applications in water resources.

\section{BACKGROUND}

\subsection{Algorithms}

A wide variety of solution methods have been formulated and applied to solve NRTP's, and summarizing them all is beyond the scope of this paper. More detailed discussions of solution methods can be found in [4, 29, 43, 49, 65]. As mentioned previously, solution methods may be classified algorithmically as either FC or SO approaches. The FC approach is sometimes called the direct substitution approach (DSA) [58, 77], the global implicit approach (GIA) 
$[49,65]$, or the one-step approach $[24,65]$. The fundamental idea of the FC approach is to solve the PDE's describing transport simultaneously with the AE's or ODE's describing the equilibrium or kinetic reaction system, respectively. Although mathematically rigorous, practical implementation of the FC approach is limited by available computer memory, since the size of the coefficient matrix in the discretized system grows as a product of the number of spatial discretization intervals and the number of species. In general, the FC approach involves solving a $N \times m$ system of nonlinear algebraic equations at each time step, where $N$ is the number nodes in the spatial mesh and $m$ is the number of chemical components.

SO approaches are commonly used in a wide variety of disciplines where transport of reacting species is important. Notable examples include the study of transport in subsurface systems $[2,21,22,25,39,45,50,53,54,59,62,66$, $71,74,76,78]$, in surface water systems [32, 48, 67], and in the atmosphere $[11,30,43,64,69]$. The basic concept of the SO approach is to solve the transport and reaction equations separately. The decoupled problem consists of $m$ transport equations, each with $N$ unknowns, and a system of $m$ reaction equations at each of the $N$ nodes. Thus, each of the subproblems has smaller memory requirements than the $\mathrm{FC}$ approach. The SO approach provides a framework that facilitates combining existing transport solvers with different reaction modules. The $\mathrm{SO}$ approach also lends itself to parallel solution, since the reaction subproblem at each node can be solved independently of the other nodes in the system. Application of SO approaches in the subsurface environment has been reviewed $[45,77]$.

Several variations of the SO approach are possible depending upon the order in which the subproblems are solved and whether or not iteration is involved. The magnitude of the error introduced by splitting depends in part on this ordering. Variants of the SO approach include the sequential SO (SSO) algorithm, the alternating SO (ASO) algorithm, and the iterative SO (ISO) algorithm.

SSO, also known as the sequential noniterative approach (SNIA) [77], is typically formulated by (1) solving the transport portion of the problem over a time interval - neglecting reactions and mass transfer, and (2) solving for the reactions over a time interval of the same length. Each portion of the solution over the time interval used may include one or more steps, e.g., the reaction step may be solved using multiple steps of an ODE solver [45].

ASO, which is closely related to Strang splitting [68] for hyperbolic PDE's, is typically formulated by (1) solving the transport portion of the problem over one-half of the time interval, (2) solving for interphase mass transfer and reaction terms over the entire time interval, and (3) solving the transport portion of the problem over the second half of the time interval. After an initial transport solution over a one-half interval of time, this algorithm reduces to 
time marching by solving for the transport and reaction steps in an alternating fashion over an entire time interval, with a half-step transport solution at points in which a solution is needed. As with the SSO algorithm, each portion of the solution may include one or more steps.

ISO, also known as the sequential iterative approach (SIA) [24, 65, 77], is typically formulated by (1) solving the transport portion of the problem over a full time interval, assuming the reaction contributions are known; (2) solving the reaction portion of the problem over a full time interval, assuming the transport contributions are known; and (3) iterating over the first two steps in the algorithm until a convergence criterion is satisfied.

\subsection{Error Analysis}

In the water resources literature, several researchers have investigated the convergence of the SO approach applied to advection-dispersion-reaction equations (ADRE's). Although work on hyperbolic problems [37, 68], has shown that time splitting can introduce numerical error for certain classes of operators $[37,68]$, convergence of the SO formulation is expected as the numerical time step approaches zero. In fact, Wheeler and Dawson [73] constructed a formal proof for the convergence of the SO approach applied to a general nonlinear ADRE.

Herzer and Kinzelbach [24] used Taylor series analysis and numerical experiments applied to a linear ADRE to show that the error introduced by time splitting in the SSO algorithm can be viewed as an additional source of numerical dispersion. Valocchi and Malmstead [70] used a heuristic analysis for a linear ADRE to show that the magnitude of splitting error for SSO is $O(\Delta t)$, where $\Delta t$ is the time step in the numerical method, which agreed with results in [24]. Numerical experiments indicated that the splitting error for the ASO algorithm could be an order of magnitude less than that of SSO for this problem. Barry et al. showed that the splitting error of the ASO algorithm

is $O\left(\Delta t^{2}\right)$ for ADRE's with linear reactions [7], but that this result does not hold for nonlinear reactions [5]. Subsequent analysis of SSO and ASO applied to linear and nonlinear ADRE's [28, 47] confirmed earlier results [5, 7, 6, 70] and highlighted the dependence of the splitting error on the magnitude of the reaction rates.

\subsection{Comparison of ISO with Other Methods}

The practical value of applying ISO to NRTP's depends upon the rate at which the splitting error is reduced. If the rate is fast enough, ISO will be compu- 
tationally efficient compared to alternative methods. Thus, formal analysis of the convergence rate of ISO applied to NRTP's and/or numerical comparisons of ISO to alternative solution algorithms are useful. We are not aware of any formal analysis on the convergence rate of the ISO algorithm applied to NRTP's, but numerical comparisons of the ISO to other SO approaches and to FC approaches have appeared in the literature over the last decade $[24,58,65,77,80]$.

Herzer and Kinzelbach [24] applied ISO to a linear ADRE and showed it to be a computationally efficient approach for reducing the error when compared to simply reducing the time step in the SSO algorithm. Yeh and Tripathi [77] analyzed computer memory requirements and estimated computational time requirements, for both the ISO and the FC approach, for two- and threedimensional NRTP's. It concluded that only ISO is viable for realistic applications. The FC approach was judged to require excessive memory and computational time. Later work on biodegradation in groundwater systems $[79,80]$ advocated the use of ASO for NRTP's with kinetic chemistry and ISO for NRTP's with equilibrium chemistry.

The most extensive work that we know of is that of Steefel and MacQuarrie [65], which considered example problems ranging from simple decay and equilibrium sorption reactions to more highly nonlinear Monod kinetic and multicomponent sorption problems. In some cases ISO gave the greatest efficiency, while in other cases SSO was more efficient. The FC approach was the least efficient from a computational viewpoint. But recent work comparing the ISO approach to the FC approach for several NRTP test cases reports that ISO is more efficient than the FC approach only for large, chemically simple problems [58]. These results seem to contradict, to some extent, the results of other researchers.

The results reported above indicate clear evidence that, at least in some cases, the convergence of the ISO algorithm is sufficiently rapid to make it computationally efficient compared to other SO approaches and the FC approach. But the results of these investigations indicate that the optimal algorithm may be problem dependent, and formal analysis of the convergence behavior of the ISO algorithm has yet to appear in the literature.

\section{ANALYSIS}

An overview of the mathematical formulation of reactive transport problems in subsurface applications is given by Rubin [56] and will not be repeated here. But in order to describe the ISO algorithm and to motivate the following analysis, we consider a multicomponent reactive transport problem of the form 


$$
\begin{aligned}
R_{f}(C) \frac{\partial C}{\partial t} & =L_{t}(C)+L_{r 1}\left(C, \omega_{s}\right) \\
\frac{\partial \omega_{s}}{\partial t} & =L_{r 2}\left(C, \omega_{s}\right)
\end{aligned}
$$

where $R_{f}(C), L_{t}(C), L_{r 1}\left(C, \omega_{s}\right), L_{r 2}\left(C, \omega_{s}\right)$ are operators. In a typical application, $L_{t}(C)$ is a linear operator containing spatial derivatives associated with advective and dispersive processes, while $R_{f}(C), L_{r 1}\left(C, \omega_{s}\right), L_{r 2}\left(C, \omega_{s}\right)$ are nonlinear operators associated with reaction processes.

\subsection{ISO Solution Algorithm}

The ISO algorithm can be expressed as a two-step procedure in which the first step solves an approximation to Eq. (1), which is typically a transport equation, and the second step solves an approximation of a local system formed by Eqs. (1)-(2), typically reaction equations at each location in space. The initial conditions for each step of the ISO algorithm is the solution from the previous time step, or the initial conditions for the first time step, although other choices are possible and may lead to a more efficient algorithm. Specifically, we consider the time interval $t \in\left[t^{0}, t^{0}+\Delta t\right]$ with initial conditions $C\left(t^{0}\right)=C^{0}$ and $\omega_{s}\left(t^{0}\right)=\omega_{s}^{0}$.

We adopt the notation $\Phi=\left(C, \omega_{s}\right)$ for the solution to the system of equations. Any stage of the iteration will move from a current approximate solution $\Phi^{c}$ to a new one $\Phi^{+}$through an intermediate step $\Phi^{1 / 2}$. In the first step, $\Phi^{1 / 2}=\left(C^{1 / 2}, \omega_{s}^{1 / 2}\right)$ is computed as the solution of

$$
\begin{aligned}
& \tilde{R}_{f} \frac{\partial C^{1 / 2}}{\partial t}=L_{t} C^{1 / 2}+\tilde{L}_{r 1}, \quad C^{1 / 2}\left(t^{0}\right)=C^{0} \\
& \omega_{s}^{1 / 2}=\omega_{s}^{c}
\end{aligned}
$$

where $\tilde{R}_{f}$ and $\tilde{L}_{r 1}$ are simple approximations. In this analysis, we use

$$
\tilde{R}_{f}=R_{f}\left(C^{c}\right) \text { and } \tilde{L}_{r 1}=L_{r 1}\left(\Phi^{c}\right)
$$

Having computed $\Phi^{1 / 2}$, then $\Phi^{+}$is the solution of

$$
\begin{aligned}
R_{f}\left(C^{+}\right) \frac{\partial C^{+}}{\partial t} & =\tilde{L}_{t}+L_{r 1}\left(C^{+}, \omega_{s}^{+}\right)=\tilde{L}_{t}+L_{r 1}\left(\Phi^{+}\right) \\
\frac{\partial \omega_{s}^{+}}{\partial t} & =L_{r 2}\left(C^{+}, \omega_{s}^{+}\right)=L_{r 2}\left(\Phi^{+}\right)
\end{aligned}
$$


with initial condition $\Phi^{+}\left(t^{0}\right)=\left(C^{0}, \omega_{s}^{0}\right)$. Here $\tilde{L}_{t}$ is an approximation. In this work, we use

$$
\tilde{L}_{t}=L_{t} C^{1 / 2}
$$

As long as the spatial discretization is fixed over the course of the ISO iterations, we can ignore the spatial variables because they have no role in the analysis and the results will hold in one-dimensional (1D), two-dimensional (2D) and three-dimensional (3D) domains.

\subsection{Preliminary Estimate}

We begin by defining the norms used throughout the analysis, then introducing a lemma which will be used twice. The norms and the lemma will be expressed in terms of a general function, $u$, and general operators $R, F_{1}$, and $F_{2}$. Approximations to $R$ and $F_{2}$ are denoted by $\tilde{R}$ and $\tilde{F}_{2}$, respectively.

If $u:[0, \Delta t] \rightarrow \mathbb{R}^{n}$ is continuous, then we define

$$
\|u\|_{\infty}=\max _{0 \leq t \leq \Delta t}\|u(t)\|
$$

where $\|\cdot\|$ is the Euclidean norm on $\mathbb{R}^{n}$.

Lemma 3.1 Assume that $u$ and $u^{*}$ are twice Lipschitz continuously differentiable $\mathbb{R}^{n}$-valued functions in $[0, \Delta t]$ and

$$
R\left(u^{*}\right) u_{t}^{*}=F_{1}\left(u^{*}\right)+F_{2}\left(u^{*}\right), \quad 0<t<\Delta t, \quad u^{*}(0)=u^{0}
$$

and

$$
\tilde{R} u_{t}=F_{1}(u)+\tilde{F}_{2}, \quad 0<t<\Delta t, \quad u(0)=u^{0} .
$$

Assume that $\left\|\tilde{R}^{1}\right\|,\left\|R^{1}\right\| \leq R_{0}{ }^{1}$ for some $R_{0}>0$. Then if $R, F_{1}$, and $F_{2}$ are Lipschitz continuous and $\Delta t$ is sufficiently small, then there is $K>0$ such that

$$
\left\|u \quad u^{*}\right\|_{\infty} \leq K \Delta t\left(\left\|F_{2}\left(u^{*}\right) \quad \tilde{F}_{2}\right\|_{\infty}+\left\|R\left(u^{*}\right) \quad \tilde{R}\right\|_{\infty}\right)
$$

Proof. Set $e=u \quad u^{*}$, so that $e_{t}=u_{t} \quad u_{t}^{*}$. Subtract Eq. (10) from Eq. (11) to obtain

$$
e_{t}=\tilde{R}^{1}\left(F_{1}(u)+\tilde{F}_{2}\right) \quad R\left(u^{*}\right)^{1}\left[F_{1}\left(u^{*}\right)+F_{2}\left(u^{*}\right)\right] .
$$


Adding and subtracting $\tilde{R}^{1}\left[F_{1}\left(u^{*}\right)+F_{2}\left(u^{*}\right)\right]$ and rearranging yields

$$
\begin{aligned}
& e_{t}=\tilde{R}^{1}\left[F_{1}(u) \quad F_{1}\left(u^{*}\right)\right]+\left(\begin{array}{lll}
\tilde{R}^{1} & R\left(u^{*}\right)^{1}
\end{array}\right) F_{1}\left(u^{*}\right) \\
& +\tilde{R}^{1}\left[\begin{array}{ll}
\tilde{F}_{2} & F_{2}\left(u^{*}\right)
\end{array}\right]+\left(\begin{array}{lll}
\tilde{R}^{1} & R\left(u^{*}\right)^{1}
\end{array}\right) F_{2}\left(u^{*}\right) .
\end{aligned}
$$

Combining the second and fourth terms in Eq. (14) yields

$$
\begin{aligned}
& e_{t}=\tilde{R}^{1}\left[F_{1}(u) \quad F_{1}\left(u^{*}\right)\right]+\frac{R\left(u^{*}\right) \quad \tilde{R}}{\tilde{R} R\left(u^{*}\right)}\left[F_{1}\left(u^{*}\right)+F_{2}\left(u^{*}\right)\right] \\
& +\tilde{R}^{1}\left[\begin{array}{ll}
\tilde{F}_{2} & F_{2}\left(u^{*}\right)
\end{array}\right] .
\end{aligned}
$$

Let $\gamma_{1}$ be the Lipschitz constant of $F_{1}$. By assumption

$$
\left\|\tilde{R}^{1}\left[F_{1}(u) \quad F_{1}\left(u^{*}\right)\right]\right\|_{\infty} \leq R_{0}{ }^{1} \gamma_{1}\|e\|_{\infty},
$$

and therefore, letting

$$
\begin{aligned}
M & =R_{0}{ }^{2}\left[\left\|F_{1}\left(u^{*}\right)\right\|_{\infty}+\left\|F_{2}\left(u^{*}\right)\right\|_{\infty}\right]\left\|R\left(u^{*}\right) \quad \tilde{R}\right\|_{\infty} \\
& +R_{0}{ }^{1}\left\|F_{2}\left(u^{*}\right) \quad \tilde{F}_{2}\right\|_{\infty},
\end{aligned}
$$

we have

$$
\left\|e_{t}(t)\right\| \leq R_{0}{ }^{1} \gamma_{1}\|e\|_{\infty}+M
$$

Hence, for all $0<t<\Delta t$,

$$
\|e(t)\|=\left\|\int_{0}^{t} e_{t}(s) d s\right\| \leq \int_{0}^{t}\left[R_{0}{ }^{1} \gamma_{1}\|e\|_{\infty}+M\right] d s .
$$

So,

$$
\|e\|_{\infty} \leq \Delta t\left(R_{0}{ }^{1} \gamma_{1}\|e\|_{\infty}+M\right)
$$

Assuming that $1 \Delta t R_{0}{ }^{1} \gamma_{1}<1 / 2$ and setting

$$
K=\max \left(R_{0}^{2}\left(\left\|F_{1}\left(u^{*}\right)\right\|_{\infty}+\left\|F_{2}\left(u^{*}\right)\right\|_{\infty}\right), R_{0}^{1}\right)
$$

completes the proof. 


\subsection{Applications of the Estimate}

We now apply the estimate given in Lemma (3.1) to the two steps of the ISO algorithm described in Eqs. (3)-(4) and (6)-(7), respectively. In each application, the general functions and operators of the lemma are replaced by specific functions and operators of the ISO step in question.

We use Lemma (3.1) to estimate $\left\|\Phi^{+} \quad \Phi^{*}\right\|_{\infty}$. We begin by showing that the error in $C^{1 / 2}$ is, up to the truncation error of the solver for Eq. (3), $O(\Delta t)$ smaller than the error in $\Phi^{c}$. We let

$$
E_{C}=C \quad C^{*} \text { and } E_{\omega}=\omega_{s} \quad \omega_{s}^{*} \text { and } E_{\Phi}=\max \left(E_{C}, E_{\omega}\right)
$$

Lemma 3.2 Assume that Eq. (5) holds. Let $C^{1 / 2}$ be the solution to Eq. (3); then there is $K^{1 / 2}>0$ such that

$$
\left\|E_{C}^{1 / 2}\right\|_{\infty} \leq K^{1 / 2} \Delta t\left\|E_{\Phi}^{c}\right\|_{\infty}
$$

Proof. We apply Lemma (3.1) to Eq. (3) with $\tilde{R}_{f}=\tilde{R}, L_{t} C=F_{1}, L_{r 1}\left(C^{*}, \omega_{s}^{*}\right)=$ $F_{2}\left(C^{*}\right)$, and $\tilde{L}_{r 1}=\tilde{F}_{2}$.

Lemma (3.2) does not imply that the solution $C^{1 / 2}$ that is computed in practice satisfies Eq. (23). If $\tau^{1 / 2}$ is the truncation error in the solution of Eq. (3) then

$$
\left\|E_{C}^{1 / 2}\right\|_{\infty} \leq K^{1 / 2} \Delta t\left\|E_{\Phi}^{c}\right\|_{\infty}+\tau^{1 / 2}
$$

In order to apply Lemma (3.1) to Eqs. (6)-(7) we must incorporate the truncation error into the $\tilde{F}_{2}$ term.

Theorem 3.3 Let $\Phi^{+}$be the solution to Eqs. (6)-(7), and let $\tau^{+}$be the truncation error in the integration. Then there is a $K^{+}$such that

$$
\left\|E_{\Phi}^{+}\right\|_{\infty} \leq K^{+} \Delta t\left(\Delta t\left\|E_{\Phi}^{c}\right\|+\tau^{1 / 2}\right)+\tau^{+}
$$

Proof. We will apply Lemma (3.1) to the complete system. Here

$$
\begin{aligned}
& R=\left(\begin{array}{cc}
R_{f} & 0 \\
0 & I
\end{array}\right), \\
& F_{1}=\left(\begin{array}{c}
L_{r 1}(\Phi) \\
L_{r 1}(\Phi)
\end{array}\right), F_{2}=\left(\begin{array}{c}
L_{t} C^{+} \\
0
\end{array}\right), \text { and } \tilde{F}_{2}=\left(\begin{array}{c}
L_{t} C^{1 / 2} \\
0
\end{array}\right) .
\end{aligned}
$$


The error in $F_{2}$ does not depend on $E_{\omega}$, in fact

$$
\left\|F_{2}\left(\Phi^{*}\right) \quad \tilde{F}_{2}\right\|_{\infty} \leq M\left\|E_{C}^{1 / 2}\right\|_{\infty} \leq M\left(K^{1 / 2} \Delta t\left\|E_{C}^{c}\right\|_{\infty}+\tau^{1 / 2}\right) .
$$

Eq. (25) follows directly from the lemma and Eq. (28).

As was the case with $\tau^{1 / 2}, \tau^{+}$reflects the truncation error of the solution. To summarize, Theorem 3.3 says that, within the truncation error of the solution methods, the error in $\Phi^{+}$should be $O\left(\Delta t^{2}\right)$ smaller than the error in $\Phi^{c}$.

\section{NUMERICAL EXPERIMENTS}

To confirm the results of the theoretical analysis, we conducted numerical investigations on a series of ODE systems and on a model PDE problem that reflects a realistic model of reactive transport in the subsurface environment.

\subsection{A Simple ODE}

We first looked at a simple ODE system

$$
\begin{aligned}
& v^{\prime}(t)=f_{1}(t) \quad 5 v(t)+v(t) w(t) \\
& w^{\prime}(t)=f_{2}(t) \quad v(t) w^{2}(t) \\
& v(0)=0 \\
& w(0)=1
\end{aligned}
$$

in which the temporal derivative term is linear in $v$. The forcing functions $f_{j}$ are constructed to make the solution of Eqs. (29)-(30)

$$
\begin{aligned}
v^{*}(t) & =\sin (t) \\
w^{*}(t) & =\exp (t)
\end{aligned}
$$

So, in the language of $\S 3.1$, we have

$$
\begin{aligned}
R_{f}(v) & =1 \\
L_{t}(v) & =5 v \\
L_{r 1}(v, w) & =f_{1}(t)+v w \\
L_{r 2}(v, w) & =f_{2}(t) \quad v(t) w^{2}(t)
\end{aligned}
$$


The initial value problem given by Eqs. (29)-(30) was solved in MATLAB using the ODE15s code [61] with relative and absolute error tolerances set at $10^{13}$.

We directed the code to output solution value at $t=\Delta t$ and used Hermite interpolation or piece-wise linear interpolation to obtain solution values for $t \in[0, \Delta t]$ to use in subsequent steps.

We report results for three iterations and six values of $\Delta t$. The initial iterate was

$$
v_{0}(t)=0, w_{0}(t)=1, \text { for } t \in(0, \Delta t) .
$$

We use the $\ell^{\infty}=$ error for $\Phi=(v, w)$,

$$
E_{i}=E\left(\Phi_{i}\right)=\left(\left|v_{i}(\Delta t) \quad v^{*}(\Delta t)\right|,\left|w_{i}(\Delta t) \quad w^{*}(\Delta t)\right|\right)
$$

where $i$ is the iteration index. In Table 1 we tabulate $E_{i}=E\left(\Phi_{i}\right)$ for $i=0,1,2$ and $\Delta t_{j}=2^{j}$ for $j=2,3, \ldots, 7$. For sufficiently small $\Delta t$, and sufficiently small truncation error, one would expect that the convergence rate

$$
Q_{i}(\Delta t)=E_{i} / E_{i}=O\left(\Delta t^{2}\right)
$$

from the results in $§ 3.3$ and hence that

$$
Q_{i}(\Delta t) / Q_{i}(2 \Delta t) \approx 0.25
$$

To observe this numerically we tabulate $E_{i}, i=0,1,2,3$ for six values of $\Delta t$ in Table 1 and $Q_{i}, i=1,2,3$ in Table 2 . In Table 3 we see the convergence predicted by the theory and Eq. (42). The interpolation error for Hermite interpolation is $O\left(\Delta t^{4}\right)$, which only begins to affect the convergence rates on the 3rd iteration.

Table 1

ODE with Hermite interpolation: Errors $E_{i}$

\begin{tabular}{|c|c|c|c|c|c|c|}
\hline$i \backslash \Delta t$ & $2.5000 \mathrm{e}-01$ & $1.2500 \mathrm{e}-01$ & $6.2500 \mathrm{e}-02$ & $3.1250 \mathrm{e}-02$ & $1.5625 \mathrm{e}-02$ & $7.8125 \mathrm{e}-03$ \\
\hline \hline 0 & $2.4740 \mathrm{e}-01$ & $1.2467 \mathrm{e}-01$ & $6.2459 \mathrm{e}-02$ & $3.1245 \mathrm{e}-02$ & $1.5624 \mathrm{e}-02$ & $7.8124 \mathrm{e}-03$ \\
\hline 1 & $1.8552 \mathrm{e}-02$ & $2.3828 \mathrm{e}-03$ & $3.0155 \mathrm{e}-04$ & $3.7921 \mathrm{e}-05$ & $4.7542 \mathrm{e}-06$ & $5.9516 \mathrm{e}-07$ \\
\hline 2 & $1.2395 \mathrm{e}-03$ & $4.3203 \mathrm{e}-05$ & $1.4199 \mathrm{e}-06$ & $4.5465 \mathrm{e}-08$ & $1.4381 \mathrm{e}-09$ & $4.5367 \mathrm{e}-11$ \\
\hline 3 & $8.4003 \mathrm{e}-05$ & $7.9894 \mathrm{e}-07$ & $6.9112 \mathrm{e}-09$ & $5.7793 \mathrm{e}-11$ & $3.1157 \mathrm{e}-13$ & $1.6342 \mathrm{e}-13$ \\
\hline
\end{tabular}


Table 2

ODE with Hermite interpolation: Ratios $Q_{i}=E_{i} / E_{i}$

\begin{tabular}{|c|c|c|c|c|c|c|}
\hline$i \backslash \Delta t$ & $2.5000 \mathrm{e}-01$ & $1.2500 \mathrm{e}-01$ & $6.2500 \mathrm{e}-02$ & $3.1250 \mathrm{e}-02$ & $1.5625 \mathrm{e}-02$ & $7.8125 \mathrm{e}-03$ \\
\hline \hline 1 & $7.4985 \mathrm{e}-02$ & $1.9112 \mathrm{e}-02$ & $4.8279 \mathrm{e}-03$ & $1.2137 \mathrm{e}-03$ & $3.0428 \mathrm{e}-04$ & $7.6181 \mathrm{e}-05$ \\
\hline 2 & $6.6811 \mathrm{e}-02$ & $1.8131 \mathrm{e}-02$ & $4.7086 \mathrm{e}-03$ & $1.1990 \mathrm{e}-03$ & $3.0250 \mathrm{e}-04$ & $7.6226 \mathrm{e}-05$ \\
\hline 3 & $6.7774 \mathrm{e}-02$ & $1.8493 \mathrm{e}-02$ & $4.8675 \mathrm{e}-03$ & $1.2711 \mathrm{e}-03$ & $2.1665 \mathrm{e}-04$ & $3.6022 \mathrm{e}-03$ \\
\hline
\end{tabular}

Table 3

ODE with Hermite interpolation: Estimated Rates $Q_{i}(\Delta t) / Q_{i}(2 \Delta t)$

\begin{tabular}{|c|c|c|c|c|c|}
\hline$i \backslash \Delta t$ & $1.2500 \mathrm{e}-01$ & $6.2500 \mathrm{e}-02$ & $3.1250 \mathrm{e}-02$ & $1.5625 \mathrm{e}-02$ & $7.8125 \mathrm{e}-03$ \\
\hline \hline 1 & $2.5488 \mathrm{e}-01$ & $2.5261 \mathrm{e}-01$ & $2.5138 \mathrm{e}-01$ & $2.5072 \mathrm{e}-01$ & $2.5036 \mathrm{e}-01$ \\
\hline 2 & $2.7138 \mathrm{e}-01$ & $2.5970 \mathrm{e}-01$ & $2.5463 \mathrm{e}-01$ & $2.5230 \mathrm{e}-01$ & $2.5199 \mathrm{e}-01$ \\
\hline 3 & $2.7286 \mathrm{e}-01$ & $2.6321 \mathrm{e}-01$ & $2.6115 \mathrm{e}-01$ & $1.7044 \mathrm{e}-01$ & $1.6627 \mathrm{e}+01$ \\
\hline
\end{tabular}

\subsection{A Simple ODE With Errors}

Here we repeat the computations from $\S 4.1$ under conditions more likely to be present in actual computations, i.e., the integration of the split equations and the operator estimates contain error. We introduce several types of error by: (1) replacing Hermite interpolation with linear interpolation; (2) increasing the tolerance of the ODE solver in both split equations; and (3) reducing the order and the accuracy of only the transport solve. The effect of any of these errors will be to increase the truncation error effects in Eqs. (24) and (25) and make Eq. (42) fail when the truncation error terms dominate the right sides of Eqs. (24) and (25).

\subsubsection{Interpolation Error}

We repeat the computations in $\$ 4.1$, except now we use linear interpolation to estimate $\tilde{L}_{r 1}$ and $\tilde{L}_{t}$ over the transport and reaction solves, respectively. The error in linear interpolation is $O\left(\Delta t^{2}\right)$, the same order as the reduction in errors. As expected, we see interpolation effects in the second iteration, shown in the second row of Table 5.

\subsubsection{Truncation Error}

We also investigate the effect on the ISO convergence rate of increased truncation error in the ODE solver. We consider the case when both steps are solved to the same tolerance, and also the case where one step is solved less accurately than the other. 
Table 4

ODE with linear interpolation: Errors $E_{i}$

\begin{tabular}{|c|c|c|c|c|c|c|}
\hline$i \backslash \Delta t$ & $2.5000 \mathrm{e}-01$ & $1.2500 \mathrm{e}-01$ & $6.2500 \mathrm{e}-02$ & $3.1250 \mathrm{e}-02$ & $1.5625 \mathrm{e}-02$ & $7.8125 \mathrm{e}-03$ \\
\hline \hline 0 & $2.4740 \mathrm{e}-01$ & $1.2467 \mathrm{e}-01$ & $6.2459 \mathrm{e}-02$ & $3.1245 \mathrm{e}-02$ & $1.5624 \mathrm{e}-02$ & $7.8124 \mathrm{e}-03$ \\
\hline 1 & $1.2823 \mathrm{e}-02$ & $1.9587 \mathrm{e}-03$ & $2.7272 \mathrm{e}-04$ & $3.6042 \mathrm{e}-05$ & $4.6343 \mathrm{e}-06$ & $5.8759 \mathrm{e}-07$ \\
\hline 2 & $2.7850 \mathrm{e}-04$ & $2.3675 \mathrm{e}-05$ & $2.0909 \mathrm{e}-06$ & $1.6016 \mathrm{e}-07$ & $1.1136 \mathrm{e}-08$ & $7.3461 \mathrm{e}-10$ \\
\hline 3 & $8.7598 \mathrm{e}-04$ & $5.3147 \mathrm{e}-05$ & $3.2574 \mathrm{e}-06$ & $2.0131 \mathrm{e}-07$ & $1.2503 \mathrm{e}-08$ & $7.7869 \mathrm{e}-10$ \\
\hline
\end{tabular}

Table 5

ODE with linear interpolation: Estimated Rates $Q_{i}(\Delta t) / Q_{i}(2 \Delta t)$

\begin{tabular}{|c|c|c|c|c|c|}
\hline$i \backslash \Delta t$ & $1.2500 \mathrm{e}-01$ & $6.2500 \mathrm{e}-02$ & $3.1250 \mathrm{e}-02$ & $1.5625 \mathrm{e}-02$ & $7.8125 \mathrm{e}-03$ \\
\hline \hline 1 & $3.0312 \mathrm{e}-01$ & $2.7793 \mathrm{e}-01$ & $2.6418 \mathrm{e}-01$ & $2.5713 \mathrm{e}-01$ & $2.5357 \mathrm{e}-01$ \\
\hline 2 & $5.5652 \mathrm{e}-01$ & $6.3427 \mathrm{e}-01$ & $5.7962 \mathrm{e}-01$ & $5.4074 \mathrm{e}-01$ & $5.2029 \mathrm{e}-01$ \\
\hline 3 & $7.1370 \mathrm{e}-01$ & $6.9401 \mathrm{e}-01$ & $8.0680 \mathrm{e}-01$ & $8.9329 \mathrm{e}-01$ & $9.4407 \mathrm{e}-01$ \\
\hline
\end{tabular}

First we repeat the computations of $\S 4.1$ using atol $=$ rtol $=10^{4}$ in the ODE solver ODE15s. The first row of Table 7 indicates that as $(\Delta t)$ is reduced, the truncation error will begin to dominate, and second-order convergence is not evident. The ratio $Q_{i}(\Delta t) / Q_{i}(2 \Delta t)$ actually increases as $(\Delta t)$ is decreased.

Table 6

ODE with solver tolerance $=10^{4}$ : Errors $E_{i}$

\begin{tabular}{|c|c|c|c|c|c|c|}
\hline$i \backslash \Delta t$ & $2.5000 \mathrm{e}-01$ & $1.2500 \mathrm{e}-01$ & $6.2500 \mathrm{e}-02$ & $3.1250 \mathrm{e}-02$ & $1.5625 \mathrm{e}-02$ & $7.8125 \mathrm{e}-03$ \\
\hline \hline 0 & $2.4740 \mathrm{e}-01$ & $1.2467 \mathrm{e}-01$ & $6.2459 \mathrm{e}-02$ & $3.1245 \mathrm{e}-02$ & $1.5624 \mathrm{e}-02$ & $7.8124 \mathrm{e}-03$ \\
\hline 1 & $1.8148 \mathrm{e}-02$ & $2.3925 \mathrm{e}-03$ & $3.3655 \mathrm{e}-04$ & $1.0494 \mathrm{e}-04$ & $4.8641 \mathrm{e}-05$ & $2.0856 \mathrm{e}-05$ \\
\hline 2 & $1.1167 \mathrm{e}-03$ & $1.8007 \mathrm{e}-04$ & $1.5880 \mathrm{e}-04$ & $1.0582 \mathrm{e}-04$ & $4.8756 \mathrm{e}-05$ & $2.0866 \mathrm{e}-05$ \\
\hline 3 & $1.3209 \mathrm{e}-04$ & $1.7829 \mathrm{e}-04$ & $1.5875 \mathrm{e}-04$ & $1.0582 \mathrm{e}-04$ & $4.8756 \mathrm{e}-05$ & $2.0866 \mathrm{e}-05$ \\
\hline
\end{tabular}

Table 7

ODE with solver tolerance $=10^{4}$ : Estimated Rates $Q_{i}(\Delta t) / Q_{i}(2 \Delta t)$

\begin{tabular}{|c|c|c|c|c|c|}
\hline$i \backslash \Delta t$ & $1.2500 \mathrm{e}-01$ & $6.2500 \mathrm{e}-02$ & $3.1250 \mathrm{e}-02$ & $1.5625 \mathrm{e}-02$ & $7.8125 \mathrm{e}-03$ \\
\hline \hline 1 & $2.6161 \mathrm{e}-01$ & $2.8079 \mathrm{e}-01$ & $6.2335 \mathrm{e}-01$ & $9.2687 \mathrm{e}-01$ & $8.5751 \mathrm{e}-01$ \\
\hline 2 & $1.2232 \mathrm{e}+00$ & $6.2691 \mathrm{e}+00$ & $2.1369 \mathrm{e}+00$ & $9.9410 \mathrm{e}-01$ & $9.9814 \mathrm{e}-01$ \\
\hline 3 & $8.3700 \mathrm{e}+00$ & $1.0097 \mathrm{e}+00$ & $1.0003 \mathrm{e}+00$ & $1.0000 \mathrm{e}+00$ & $1.0000 \mathrm{e}+00$ \\
\hline
\end{tabular}

Fully coupled solutions to NRTP's are typically implemented using low-order finite difference or finite element methods which are second-order accurate in space and time. Although typical ISO implementations solve the reaction step using methods which are higher order in time, the transport step is often solved using methods that are lower order in time and often use fixed time 
steps. To model this scenario, we repeat the calculations of $\S 4.1$ but solve the first step of the ISO algorithm (Eq. 3) using a second-order accurate integration method and adjust the solver tolerance such that the solution over the timestep $\Delta t$ is completed in only one or two substeps. The second step of the ISO algorithm (Eqs. 6-7) is computed using the same adaptive time stepping and tolerance used previously in $\S 4.1$. The rates in Table 9 show that we deviate from the second-order convergence, but the deviation appears smaller as $(\Delta t)$ is decreased. This behavior was also observed in Table (5), where we reduced the interpolation accuracy.

Table 8

ODE with one low-order substep: $E_{i}$

\begin{tabular}{|c|c|c|c|c|c|c|}
\hline$i \backslash \Delta t$ & $2.5000 \mathrm{e}-01$ & $1.2500 \mathrm{e}-01$ & $6.2500 \mathrm{e}-02$ & $3.1250 \mathrm{e}-02$ & $1.5625 \mathrm{e}-02$ & $7.8125 \mathrm{e}-03$ \\
\hline \hline 0 & $2.4740 \mathrm{e}-01$ & $1.2467 \mathrm{e}-01$ & $6.2459 \mathrm{e}-02$ & $3.1245 \mathrm{e}-02$ & $1.5624 \mathrm{e}-02$ & $7.8124 \mathrm{e}-03$ \\
\hline 1 & $1.8446 \mathrm{e}-02$ & $2.8784 \mathrm{e}-03$ & $4.2037 \mathrm{e}-04$ & $5.7744 \mathrm{e}-05$ & $7.6062 \mathrm{e}-06$ & $9.7747 \mathrm{e}-07$ \\
\hline 2 & $3.7074 \mathrm{e}-04$ & $5.3095 \mathrm{e}-05$ & $5.4449 \mathrm{e}-06$ & $4.4909 \mathrm{e}-07$ & $3.2553 \mathrm{e}-08$ & $2.1970 \mathrm{e}-09$ \\
\hline 3 & $1.4908 \mathrm{e}-03$ & $1.1341 \mathrm{e}-04$ & $8.1405 \mathrm{e}-06$ & $5.5323 \mathrm{e}-07$ & $3.6211 \mathrm{e}-08$ & $2.3185 \mathrm{e}-09$ \\
\hline
\end{tabular}

Table 9

ODE with one low-order substep: Estimated Rates $Q_{i}(\Delta t) / Q_{i}(2 \Delta t)$

\begin{tabular}{|c|c|c|c|c|c|}
\hline$i \backslash \Delta t$ & $1.2500 \mathrm{e}-01$ & $6.2500 \mathrm{e}-02$ & $3.1250 \mathrm{e}-02$ & $1.5625 \mathrm{e}-02$ & $7.8125 \mathrm{e}-03$ \\
\hline \hline 1 & $3.0966 \mathrm{e}-01$ & $2.9151 \mathrm{e}-01$ & $2.7460 \mathrm{e}-01$ & $2.6341 \mathrm{e}-01$ & $2.5701 \mathrm{e}-01$ \\
\hline 2 & $9.1776 \mathrm{e}-01$ & $7.0221 \mathrm{e}-01$ & $6.0044 \mathrm{e}-01$ & $5.5029 \mathrm{e}-01$ & $5.2517 \mathrm{e}-01$ \\
\hline 3 & $5.3118 \mathrm{e}-01$ & $6.9994 \mathrm{e}-01$ & $8.2396 \mathrm{e}-01$ & $9.0297 \mathrm{e}-01$ & $9.4873 \mathrm{e}-01$ \\
\hline
\end{tabular}

\subsection{A More Complicated ODE}

Many subsurface transport modeling efforts employ equilibrium mass transfer relationships that lead to an $R_{f}$ that is not Lipschitz continuous near $C=0$ [51]. The theoretical analysis assumes that $R_{f}$ is Lipschitz, but one might ask if this is a necessary condition. To investigate the sensitivity of the analysis to the Lipschitz assumption, we considered ODE systems with $R_{f}(v)=1 / \sqrt{v}$ and $R_{f}(v)=1 /\left(1+v^{2}\right)$. In the first case, the $R_{f}$ has a form that arises in subsurface modeling applications and for which the assumption of Lipschitz continuity is violated. The second form is sufficiently smooth. One might expect that the first form degrades the ISO convergence rate while the second does not. The results for $R_{f}(v)=1 / \sqrt{v}$ in Tables $10-11$ and $R_{f}(v)=1 /\left(1+v^{2}\right)$ in Tables 12-13 confirm this expectation. 
Table 10

ODE with $R_{f}(v)=1 / \sqrt{v}$ : Errors $E_{i}$

\begin{tabular}{|c|c|c|c|c|c|c|}
\hline$i \backslash \Delta t$ & $2.5000 \mathrm{e}-01$ & $1.2500 \mathrm{e}-01$ & $6.2500 \mathrm{e}-02$ & $3.1250 \mathrm{e}-02$ & $1.5625 \mathrm{e}-02$ & $7.8125 \mathrm{e}-03$ \\
\hline \hline 0 & $2.4740 \mathrm{e}-01$ & $1.2467 \mathrm{e}-01$ & $6.2459 \mathrm{e}-02$ & $3.1245 \mathrm{e}-02$ & $1.5624 \mathrm{e}-02$ & $7.8124 \mathrm{e}-03$ \\
\hline 1 & $1.4964 \mathrm{e}-02$ & $3.6734 \mathrm{e}-03$ & $8.1460 \mathrm{e}-04$ & $1.6783 \mathrm{e}-04$ & $3.2646 \mathrm{e}-05$ & $5.7186 \mathrm{e}-06$ \\
\hline 2 & $1.9867 \mathrm{e}-04$ & $2.7798 \mathrm{e}-05$ & $3.8206 \mathrm{e}-06$ & $5.0851 \mathrm{e}-06$ & $6.3568 \mathrm{e}-07$ & $7.9464 \mathrm{e}-08$ \\
\hline 3 & $5.1079 \mathrm{e}-06$ & $5.6116 \mathrm{e}-06$ & $6.1460 \mathrm{e}-06$ & $5.0852 \mathrm{e}-06$ & $6.3569 \mathrm{e}-07$ & $7.9465 \mathrm{e}-08$ \\
\hline
\end{tabular}

Table 11

ODE with $R_{f}(v)=1 / \sqrt{v}$ : Estimated Rates $Q_{i}(\Delta t) / Q_{i}(2 \Delta t)$

\begin{tabular}{|c|c|c|c|c|c|}
\hline$i \backslash \Delta t$ & $1.2500 \mathrm{e}-01$ & $6.2500 \mathrm{e}-02$ & $3.1250 \mathrm{e}-02$ & $1.5625 \mathrm{e}-02$ & $7.8125 \mathrm{e}-03$ \\
\hline \hline 1 & $4.8714 \mathrm{e}-01$ & $4.4265 \mathrm{e}-01$ & $4.1186 \mathrm{e}-01$ & $3.8898 \mathrm{e}-01$ & $3.5033 \mathrm{e}-01$ \\
\hline 2 & $5.6996 \mathrm{e}-01$ & $6.1980 \mathrm{e}-01$ & $6.4600 \mathrm{e}+00$ & $6.4267 \mathrm{e}-01$ & $7.1363 \mathrm{e}-01$ \\
\hline 3 & $7.8519 \mathrm{e}+00$ & $7.9684 \mathrm{e}+00$ & $6.2166 \mathrm{e}-01$ & $9.9999 \mathrm{e}-01$ & $9.9999 \mathrm{e}-01$ \\
\hline
\end{tabular}

Table 12

ODE with $R_{f}(v)=1 /\left(1+v^{2}\right)$ : Errors $E_{i}$

\begin{tabular}{|c|c|c|c|c|c|c|}
\hline$i \backslash \Delta t$ & $2.5000 \mathrm{e}-01$ & $1.2500 \mathrm{e}-01$ & $6.2500 \mathrm{e}-02$ & $3.1250 \mathrm{e}-02$ & $1.5625 \mathrm{e}-02$ & $7.8125 \mathrm{e}-03$ \\
\hline \hline 0 & $2.4740 \mathrm{e}-01$ & $1.2467 \mathrm{e}-01$ & $6.2459 \mathrm{e}-02$ & $3.1245 \mathrm{e}-02$ & $1.5624 \mathrm{e}-02$ & $7.8124 \mathrm{e}-03$ \\
\hline 1 & $2.4247 \mathrm{e}-02$ & $2.6466 \mathrm{e}-03$ & $3.1588 \mathrm{e}-04$ & $3.8757 \mathrm{e}-05$ & $4.8048 \mathrm{e}-06$ & $5.9827 \mathrm{e}-07$ \\
\hline 2 & $2.7184 \mathrm{e}-03$ & $5.9384 \mathrm{e}-05$ & $1.6382 \mathrm{e}-06$ & $4.8655 \mathrm{e}-08$ & $1.4864 \mathrm{e}-09$ & $4.6109 \mathrm{e}-11$ \\
\hline 3 & $2.9909 \mathrm{e}-04$ & $1.3457 \mathrm{e}-06$ & $8.7217 \mathrm{e}-09$ & $6.4374 \mathrm{e}-11$ & $3.3619 \mathrm{e}-13$ & $1.6334 \mathrm{e}-13$ \\
\hline
\end{tabular}

Table 13

ODE with $R_{f}(v)=1 /\left(1+v^{2}\right)$ : Estimated Rates $Q_{i}(\Delta t) / Q_{i}(2 \Delta t)$

\begin{tabular}{|c|c|c|c|c|c|}
\hline$i \backslash \Delta t$ & $1.2500 \mathrm{e}-01$ & $6.2500 \mathrm{e}-02$ & $3.1250 \mathrm{e}-02$ & $1.5625 \mathrm{e}-02$ & $7.8125 \mathrm{e}-03$ \\
\hline \hline 1 & $2.1660 \mathrm{e}-01$ & $2.3824 \mathrm{e}-01$ & $2.4528 \mathrm{e}-01$ & $2.4791 \mathrm{e}-01$ & $2.4902 \mathrm{e}-01$ \\
\hline 2 & $2.0014 \mathrm{e}-01$ & $2.3113 \mathrm{e}-01$ & $2.4206 \mathrm{e}-01$ & $2.4642 \mathrm{e}-01$ & $2.4914 \mathrm{e}-01$ \\
\hline 3 & $2.0596 \mathrm{e}-01$ & $2.3493 \mathrm{e}-01$ & $2.4851 \mathrm{e}-01$ & $1.7095 \mathrm{e}-01$ & $1.5662 \mathrm{e}+01$ \\
\hline
\end{tabular}

\subsection{A Nonlinear Reactive Transport Problem}

The previous numerical experiments using ODE's provide some insight into the convergence behavior of the ISO algorithm but do not guarantee that the same behavior will be observed in more realistic situations. Thus, we also consider the numerical solution of a model NRTP of the form described by Eqs. (1)-(2). 


\subsubsection{Model Formulation}

For convenience, we consider a model problem introduced in [29], which describes solute transport, reaction, and interphase mass transfer in a porous medium system. Mass transfer occurs between a fluid phase and two types of solid phase - a fraction that achieves equilibrium quickly and a fraction that achieves equilibrium slowly. Transformation reactions are considered to be of a general form and may be linear or nonlinear. Subsets of this general model occur routinely in application, and because of this it makes a good test problem for the solution issues of concern in this work. Because the major aspects

of this work do not depend upon the spatial dimensionality of the system, we will restrict our efforts to one spatial dimension. We also limit ourselves to the case of transport with a known uniform flow field. This subset of the governing equations describing 1D transport in a uniform flow field is often employed for problems such as solute transport through soil columns, e.g. [17, 38, 20], fixed bed reactors, e.g. [3, 34, 46], and in many field-scale investigations, e.g. $[1,55,60]$.

We formulate this model as

$$
\begin{aligned}
& \frac{\partial C}{\partial t}+\frac{\rho_{b}}{\theta} \frac{\partial \omega_{f}}{\partial t}=D_{x} \frac{\partial^{2} C}{\partial x^{2}} \quad v_{x} \frac{\partial C}{\partial x} \quad k_{a} C^{r_{a}} \\
& \frac{\rho_{b}}{\theta}\left[\alpha\left(\begin{array}{ll}
\omega_{s}^{e} & \left.\omega_{s}\right)+k_{f} \omega_{f}^{r_{f}}
\end{array}\right] \text {, in }\left[0, L_{x}\right] \times\left[0, t_{s}\right]\right. \\
& \frac{\partial \omega_{s}}{\partial t}=\alpha\left(\omega_{s}^{e} \quad \omega_{s}\right) \quad k_{s} \omega_{s}^{r_{s}}, \text { in }\left[0, L_{x}\right] \times\left[0, t_{s}\right]
\end{aligned}
$$

subject to boundary conditions

$$
\begin{aligned}
C(0, t) & =C_{0} \\
\frac{\partial C}{\partial x}\left(L_{x}, t\right) & =0
\end{aligned}
$$

and initial conditions

$$
\begin{aligned}
& C(x, 0)=\sin \left(\frac{\pi x}{L_{x}}\right) \\
& \omega_{s}(x, 0)=0
\end{aligned}
$$

where $\left[0, L_{x}\right] \subset \mathbb{R}^{1}$ is the spatial domain; $\left[0, t_{s}\right]$ is the temporal domain; $x$ and $t$ are the spatial and temporal coordinates, respectively; $C$ is an aqueous phase solute concentration; $t$ is time; $D_{x}$ is a hydrodynamic dispersion coefficient; $v_{x}$ is a mean pore velocity; $k_{a}, k_{f}$, and $k_{s}$ are reaction rate constants for the aqueous-phase, the rapidly sorbing solid phase, and the slowly sorbing solid 
phase, respectively; $r_{a}, r_{f}$, and $r_{s}$ are reaction order exponents for the aqueous phase, the rapidly sorbing solid phase, and the slowly sorbing solid phase, respectively; $\rho_{b}$ is a solid-phase bulk density; $\alpha$ is a first-order mass transfer rate coefficient for mass transfer between the aqueous phase and the slowly sorbing solid phase; $\theta$ is porosity; $\omega$ is a mass averaged solid-phase solute concentration, i.e., mass fraction; $\omega_{f}$ and $\omega_{s}$ are solid-phase solute mass fractions for the rapidly sorbing and slowly sorbing solid phase fractions, respectively; and $\omega^{e}, \omega_{f}^{e}$ and $\omega_{s}^{e}$ are equilibrium solute mass fractions for the total, the rapidly sorbing and slowly sorbing solid phase fraction, respectively, in equilibrium with the aqueous-phase solute concentration $C^{e}$.

Equilibrium mass transfer between the fluid and solid phases is described by a combination of Freundlich equilibrium expressions

$$
\begin{aligned}
& \omega^{e}=\omega_{f}^{e}+\omega_{s}^{e} \\
& \omega^{e}=K_{f}\left(C^{e}\right)^{n_{f}}+K_{s}\left(C^{e}\right)^{n_{s}}
\end{aligned}
$$

where $K_{f}$ and $K_{s}$ are Freundlich sorption capacity coefficients for the rapidly sorbing and slowly sorbing solid-phase fraction, respectively; and $n_{f}$ and $n_{s}$ are Freundlich exponents for the rapidly sorbing and slowly sorbing fraction, respectively.

The formulation summarized by Eqs. (43)-(50) is a coupled system of nonlinear PDE's in two primary dependent variables, $C$ and $\omega_{s}$, if $\omega_{f}$ is expressed as a function of $C$ by invoking the assumption of local equilibrium. Eq. 43 is frequently written in non-conservative form (e.g. [14, 23]) by assuming local equilibrium for the fast sites and using the chain rule to yield

$$
\begin{aligned}
R_{f} \frac{\partial C}{\partial t}= & D_{x} \frac{\partial^{2} C}{\partial x^{2}} \quad v_{x} \frac{\partial C}{\partial x} \quad k_{a} C^{r_{a}} \\
& \frac{\rho_{b}}{\theta}\left[\begin{array}{ll}
\alpha\left(\omega_{s}^{e}\right. & \left.\omega_{s}\right)+k_{f} \omega_{f}^{r_{f}}
\end{array}\right], \text { in } \Omega \times\left[0, t_{s}\right] \\
R_{f}= & 1+\frac{\rho_{b}}{\theta} \frac{\partial \omega_{f}}{\partial C}
\end{aligned}
$$

where $R_{f}$ is termed the retardation factor. We use this form of the equation in this work.

In terms of the operator notation introduced $\S 3$, we have

$$
\begin{aligned}
R_{f}(C) & =1+\frac{\rho_{b}}{\theta} K_{f} n_{f} C^{n_{f}} \quad 1 \\
L_{t}(C) & =D_{x} \frac{\partial^{2} C}{\partial x^{2}} \quad v_{x} \frac{\partial C}{\partial x}
\end{aligned}
$$




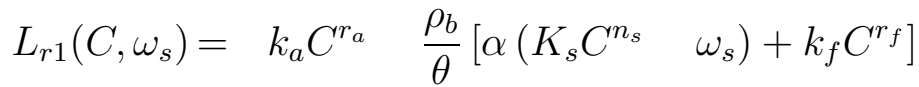

$$
\begin{aligned}
& L_{r 2}\left(C, \omega_{s}\right)=\alpha\left(K_{s} C^{n_{s}} \quad \omega_{s}\right) \quad k_{s} \omega_{s}^{r_{s}}
\end{aligned}
$$

\subsubsection{Numerical Solution}

We implemented the ISO algorithm using an approach in which the transport and reaction substeps are both solved using an ODE solver with formal error control. The transport substep is solved using a method of lines (MOL) approach in which the spatial derivatives are approximated using a centered finite difference to produce an ODE system for the concentrations at the nodes. This ODE system is integrated over $[0, \Delta t]$ to a prescribed error tolerance using the stiff ODE solver ODE15s. The reaction substep consists of solving a local reaction problem over $[0, \Delta t]$ at each node, again using ODE15s to produce a solution with a prescribed level of error.

We implemented several methods for computing the reaction operator estimate $\tilde{L}_{r 1}$ used in the transport substep, and the transport operator estimate $\tilde{L}_{t}$ used in the reaction substep. In the simplest approach these estimates were held constant over the respective substeps. Before the transport solve, the reaction operator estimate $L_{r 1}$ is computed using the current approximate solution. It is then kept constant during the transport solve. Before the reaction solve, the transport operator estimate $L_{t}$ is computed using the currrent approximate solution. It is then kept constant during the reaction solve. We implemented two approaches which represented $\tilde{L}_{t}$ and $\tilde{L}_{r 1}$ as functions of time. One approach uses linear interpolation between the current approximate solution and the solution at the begining of the time step. Another approach uses Hermite interpolation.

Based on the results in $\S 4.3$ for the non-Lipschitz $R_{f}$, we considered a technique that uses a smooth approximation of the Freundlich isotherm. The approach approximates $\omega_{f}^{e}=K_{f}\left(C^{e}\right)^{n_{f}}$ with a cubic spline, using about 1000 points to cover the interval $C \in\left[0, C_{0}\right]$. Then $R_{f}$ was computed using the derivative of the spline representation of $\omega_{f}^{e}$.

\subsubsection{Numerical Experiments}

In this instance no analytical solution is available. Therefore, our exact solution is a numerical one computed with a MOL approach using the stiff ODE solver ODE15s. We selected this approach because it allows us to control the temporal error. We use this exact solution to compute the error in the ISO solution at each iteration. We compare the convergence over a single time step.

Table 14 shows the parameter set used in the model problem. 
Table 14

Model Parameters

\begin{tabular}{|c|c|c|c|c|c|c|c|c|c|}
\hline$D_{x}$ & $v_{x}$ & $\rho_{b}$ & $\theta$ & $\alpha$ & $K_{f}$ & $K_{s}$ & $k_{a}$ & $k_{f}$ & $k_{s}$ \\
\hline 0.01 & 1 & 1.59 & 0.4 & 0.1 & 0.126 & 0.252 & 1 & 1 & 1 \\
\hline \hline$n_{f}$ & $n_{s}$ & $r_{a}$ & $r_{f}$ & $r_{s}$ & $L_{x}$ & $t_{s}$ & $C_{0}$ & $C^{0}$ & $\omega_{s}^{0}$ \\
\hline 0.7 & 0.7 & 1 & 1 & 1 & 1 & 1 & 1 & 0 & 0 \\
\hline
\end{tabular}

Numerical experiments were conducted for a single time step over a wide range of spatial and temporal discretizations. We considered the effect of interpolation accuracy for $\tilde{L}_{t}$ and $\tilde{L}_{r_{1}}$ on convergence by using constant, linear, and Hermite interpolation. We observed results similar to those for the simple ODE problem, so they will not be shown here. In summary, we found that constant and linear interpolation both degraded the convergence rate to $O(\Delta t)$, while Hermite interpolation of the operator estimates preserved the $O\left(\Delta t^{2}\right)$ convergence rate predicted by the analysis.

Tables 15-16 and 17-18 show the errors and convergence rate for the unsplined and splined $R_{f}$ approaches, respectively, for 50 cell spatial discretization. Hermite interpolation was used in both approaches, and $\Delta t=1 / 2^{k}$ for $k=$ $6,7, \ldots, 11$. The exact solution and the substeps of the ISO solution were solved in MATLAB using the ODE15s code with relative and absolute error tolerances set at $10^{13}$. We observe that using the splined $R_{f}$ approach preserves the $O\left(\Delta t^{2}\right)$ convergence rate.

Table 15

Model PDE with unsplined $R_{f}$ approach: Errors $E_{i}$

\begin{tabular}{|c|c|c|c|c|c|c|}
\hline$i \backslash \Delta t$ & $1.5625 \mathrm{e}-02$ & $7.8125 \mathrm{e}-03$ & $3.9062 \mathrm{e}-03$ & $1.9531 \mathrm{e}-03$ & $9.7656 \mathrm{e}-04$ & $4.8828 \mathrm{e}-04$ \\
\hline \hline 0 & $1.3289 \mathrm{e}-01$ & $6.8916 \mathrm{e}-02$ & $3.4932 \mathrm{e}-02$ & $1.7561 \mathrm{e}-02$ & $8.8004 \mathrm{e}-03$ & $4.4048 \mathrm{e}-03$ \\
\hline 1 & $6.1164 \mathrm{e}-03$ & $1.1270 \mathrm{e}-03$ & $2.1160 \mathrm{e}-04$ & $4.1161 \mathrm{e}-05$ & $8.1896 \mathrm{e}-06$ & $1.6388 \mathrm{e}-06$ \\
\hline 2 & $1.6587 \mathrm{e}-03$ & $2.4611 \mathrm{e}-04$ & $3.6634 \mathrm{e}-05$ & $6.7019 \mathrm{e}-06$ & $1.2379 \mathrm{e}-06$ & $2.2518 \mathrm{e}-07$ \\
\hline 3 & $1.6622 \mathrm{e}-03$ & $2.4636 \mathrm{e}-04$ & $3.7587 \mathrm{e}-05$ & $6.7780 \mathrm{e}-06$ & $1.2441 \mathrm{e}-06$ & $2.2566 \mathrm{e}-07$ \\
\hline
\end{tabular}

Table 16

Model PDE with unsplined $R_{f}$ approach: Estimated Rates $Q_{i}(\Delta t) / Q_{i}(2 \Delta t)$

\begin{tabular}{|c|c|c|c|c|c|}
\hline$i \backslash \Delta t$ & $7.8125 \mathrm{e}-03$ & $3.9062 \mathrm{e}-03$ & $1.9531 \mathrm{e}-03$ & $9.7656 \mathrm{e}-04$ & $4.8828 \mathrm{e}-04$ \\
\hline \hline 1 & $3.5530 \mathrm{e}-01$ & $3.7041 \mathrm{e}-01$ & $3.8696 \mathrm{e}-01$ & $3.9702 \mathrm{e}-01$ & $3.9980 \mathrm{e}-01$ \\
\hline 2 & $8.0524 \mathrm{e}-01$ & $7.9281 \mathrm{e}-01$ & $9.4046 \mathrm{e}-01$ & $9.2836 \mathrm{e}-01$ & $9.0900 \mathrm{e}-01$ \\
\hline 3 & $9.9892 \mathrm{e}-01$ & $1.0250 \mathrm{e}+00$ & $9.8570 \mathrm{e}-01$ & $9.9368 \mathrm{e}-01$ & $9.9723 \mathrm{e}-01$ \\
\hline
\end{tabular}


Table 17

Model PDE with splined $R_{f}$ approach: Errors $E_{i}$

\begin{tabular}{|c|c|c|c|c|c|c|}
\hline$i \backslash \Delta t$ & $1.5625 \mathrm{e}-02$ & $7.8125 \mathrm{e}-03$ & $3.9062 \mathrm{e}-03$ & $1.9531 \mathrm{e}-03$ & $9.7656 \mathrm{e}-04$ & $4.8828 \mathrm{e}-04$ \\
\hline \hline 0 & $1.3289 \mathrm{e}-01$ & $6.8916 \mathrm{e}-02$ & $3.4932 \mathrm{e}-02$ & $1.7561 \mathrm{e}-02$ & $8.8004 \mathrm{e}-03$ & $4.4048 \mathrm{e}-03$ \\
\hline 1 & $1.4244 \mathrm{e}-03$ & $2.2490 \mathrm{e}-04$ & $3.1539 \mathrm{e}-05$ & $4.1739 \mathrm{e}-06$ & $5.3679 \mathrm{e}-07$ & $6.8058 \mathrm{e}-08$ \\
\hline 2 & $1.6587 \mathrm{e}-03$ & $2.4611 \mathrm{e}-04$ & $3.5803 \mathrm{e}-05$ & $4.8263 \mathrm{e}-06$ & $6.2645 \mathrm{e}-07$ & $7.9793 \mathrm{e}-08$ \\
\hline 3 & $1.6622 \mathrm{e}-03$ & $2.4636 \mathrm{e}-04$ & $3.5812 \mathrm{e}-05$ & $4.8266 \mathrm{e}-06$ & $6.2646 \mathrm{e}-07$ & $7.9794 \mathrm{e}-08$ \\
\hline
\end{tabular}

Table 18

Model PDE with splined $R_{f}$ approach: Estimated Rates $Q_{i}(\Delta t) / Q_{i}(2 \Delta t)$

\begin{tabular}{|c|c|c|c|c|c|}
\hline$i \backslash \Delta t$ & $7.8125 \mathrm{e}-03$ & $3.9062 \mathrm{e}-03$ & $1.9531 \mathrm{e}-03$ & $9.7656 \mathrm{e}-04$ & $4.8828 \mathrm{e}-04$ \\
\hline \hline 1 & $3.0445 \mathrm{e}-01$ & $2.7666 \mathrm{e}-01$ & $2.6326 \mathrm{e}-01$ & $2.5662 \mathrm{e}-01$ & $2.5331 \mathrm{e}-01$ \\
\hline 2 & $9.3971 \mathrm{e}-01$ & $1.0374 \mathrm{e}+00$ & $1.0186 \mathrm{e}+00$ & $1.0093 \mathrm{e}+00$ & $1.0046 \mathrm{e}+00$ \\
\hline 3 & $9.9892 \mathrm{e}-01$ & $9.9924 \mathrm{e}-01$ & $9.9980 \mathrm{e}-01$ & $9.9995 \mathrm{e}-01$ & $9.9999 \mathrm{e}-01$ \\
\hline
\end{tabular}

\section{DISCUSSION}

The analysis demonstrates that under fairly reasonable smoothness assumptions, one can show that the theoretical convergence rate for ISO applied to our model NRTP's is $O\left(\Delta t^{2}\right)$, where $\Delta t$ is the time step of the numerical method. This result is independent of the class of spatial discretization employed and is valid in $1 \mathrm{D}, 2 \mathrm{D}$, or $3 \mathrm{D}$ domains. The theory does assume that the relevant temporal integration of the split equations can be done as accurately as needed. Obviously, this assumption must be relaxed in practice and may result in slower convergence in real applications.

Numerical experiments on a simple ODE system indicate that several factors may reduce the convergence rate of ISO in practice. We observed that reducing the accuracy used in the integration of the split equations has an adverse effect on the order of convergence. We also observed that reducing the accuracy of the interpolation used in the operator estimates can lower the convergence rate.

Numerical experiments using the simple ODE also demonstrate that in order to preserve the second-order convergence of the ISO algorithm, one must have accurate estimates for the reaction and transport operators in the transport solve and reaction solve, respectively. Our results for the simple ODE showed that linear interpolation effects were seen after one iteration. Results not tabulated here showed that using constants for these operator estimates completely destroyed the second-order convergence for the simple ODE. Since using a constant approximation of the these operators is a typical approach 
$[42,59,71]$, it has significant implications for solving realistic problems.

Numerical experiments using an ODE with a model $R_{f}$ term lend insight into the importance of the Lipschitz continuity assumption used in the analysis. The analysis treats Lipschitz continuity as a sufficient condition, but the numerical experiments indicate that it may be a necessary condition. This has significant practical implications for subsurface transport investigations, since the Freundlich model for sorption equilibrium often used in subsurface transport simulations results in an $R_{f}$ which is non-Lipschitz for very low concentrations. The Freundlich model does not reduce to a linear relationship between $C$ and $\omega$ at low fluid-phase concentrations. Instead, $\partial \omega / \partial C$ tends to infinity as the fluid phase solute concentration approaches zero.

The results of the numerical experiments on the model NRTP show that one can implement the ISO algorithm in a manner that preserves the second-order convergence predicted by the theory. Simple constant or linear approximations for the transport and reaction operator estimates destroy the second-order convergence rate, while Hermite interpolation along with a smooth approximation for the retardation factor preserve second-order convergence. We stress that we looked at convergence, and we have not systematically investigated the computational efficiency of this approach. If the work for each iteration of the ISO method is excessive, it may not be competitive with other SO approaches or with the best FC approaches. This is an area which deserves further research.

\section{CONCLUSIONS}

Based upon the analysis and numerical experiments, We draw several conclusions regarding the use of the ISO algorithm for NRTP's:

(1) Theoretical analysis shows that, given certain assumptions regarding smoothness, the rate of convergence for ISO solution of NRTP's is $O\left(\Delta t^{2}\right)$.

(2) Numerical experiments using a simple ODE system indicate that the theoretical convergence rate can be degraded by discretization errors introduced into the numerical solution and by coarse estimates of the reaction and transport operators. Experiments on ODE models also imply that Lipschitz continuity is an important assumption in practice.

(3) Numerical experiments on a model PDE system using typical finite difference implementations of the ISO approach (i.e., with constant and linear estimates of the reaction and transport operators) and using the Freundlich sorption equilibrium model agree with the results of ODE experiments.

(4) Our results show that by using Hermite interpolation to compute the 
transport and reaction operator estimates, and using a smooth approximation to the Freundlich sorption model, one can obtain the theoretical rate of convergence.

(5) Additional research on the computational efficiency of the the ISO approach introduced here is needed before it is proposed for routine subsurface simulation projects.

\section{Acknowledgements}

The UNC aspects of this work have been supported by the National Institute of Environmental Health Sciences (NIEHS) grant 5 P42 ES05948-02 and the National Science Foundation (NSF) grant DMS-0112653. The work at NCSU has been supported by NSF grants DMS-0070641 and DMS-0112542, and the US Army Research Office (ARO) grant DAAD19-99-1-0186. Computational support was provided by the North Carolina Supercomputer Center and from Cray Research, Inc.

\section{References}

[1] R.H. Abrams and K. Loague. A compartmentalized solute transport model for redox zones in contaminated aquifers, 2. Field-scale simulations. Water Resources Research, 36(8):2015-2029, 2000.

[2] C. A. J. Appelo and A. Willemsen. Geochemical calculations and observations on salt water intrusions, I. A combined geochemical/mixing cell model. Journal of Hydrology, 94:313-330, 1987.

[3] A. K. Avci, D. L. Trimm, and Z. I. Onsan. Heterogeneous reactor modeling for simulation of catalytic oxidation and steam reforming of methane. Chemical Engineering Science, 56(2):641-649, 2001.

[4] D. A. Barry. Supercomputers and their use in modeling subsurface solute transport. Reviews of Geophysics, 38(3):277-295, 1990.

[5] D. A. Barry, C. T. Miller, P. J. Culligan, and K. Bajracharya. Split operator methods for reactive chemical transport in groundwater. In Proceeding of the International Conference on Modeling and Simulation 1995: MODSIM95, pages 125-132, New South Wales, 1995. Modeling and Simulation Society of Australia, Inc.

[6] D. A. Barry, C. T. Miller, P. J. Culligan, and K. Bajracharya. Analysis of split operator methods for nonlinear and multispecies groundwater chemical transport models. Mathematics and Computers in Simulation, 43:331-341, 1997.

[7] D. A. Barry, C. T. Miller, and P. J. Culligan-Hensley. Temporal discretization errors in non-iterative split-operator approaches to solving chemical 
reaction/groundwater transport models. Journal of Contaminant Hydrology, 22(1/2):1-17, 1996.

[8] D.A. Barry, K. Bajracharya, M. Crapper, H. Prommer, and C.J. Cunningham. Comparison of split-operator methods for solving coupled chemical non-equilibrium reaction/groundwater transport models. Mathematics and Computers in Simulation, 53:113-127, 2000.

[9] I. Bey, D.J. Jacob, R.M. Yantosca, J.A. Logan, B.D. Field, A.M. Fiore, Q.B. Li, H.G.Y. Liu, L.J. Mickley, and M.G. Schultz. Global modeling of tropospheric chemistry with assimilated meteorology: Model description and evaluation. Journal of Geophysical Research-Atmospheres, 106(D19): 23073-3095, 2001.

[10] D. Bhuyan, L. W. Lake, and G. A. Pope. Mathematical modeling of high-pH chemical flooding. SPE Reservoir Engineering, 5:213-220, 1990.

[11] J.G. Blom and J.G. Verwer. A comparison of integration methods for atmospheric transport-chemistry problems. Journal of Computational and Applied Mathematics, 126(1-2):381-396, 2000.

[12] M. Blunt, F. J. Fayers, and F. M. Orr. Carbon-dioxide in enhanced oilrecovery. Energy Conversion and Management, 34(9-1):1197-1204, 1992.

[13] R. C. Borden, P. B. Bedient, M. D. Lee, C. H. Ward, and J. T. Wilson. Transport of dissolved hydrocarbons influenced by oxygen-limited biodegradation, 2. Field application. Water Resources Research, 22(13): 1983-1990, 1986.

[14] M. L. Brusseau and P. S. C. Rao. Sorption nonideality during organic contaminant transport in porous media. Critical Reviews in Environmental Control, 19(1):33-99, 1989.

[15] M. A. Celia, J. S. Kindred, and I. Herrera. Contaminant transport and biodegradation 1. A numerical model for reactive transport in porous media. Water Resources Research, 25(6):1141-1148, 1989.

[16] H. P. Cheng and G. T. Yeh. Development and demonstrative application of a 3-D numerical model of subsurface flow, heat transfer, and reactive chemical transport: 3DHYDROGEOCHEM. Journal of Contaminant Hydrology, 34(1-2):47-83, 1998.

[17] C. V. Chrysikopoulos, P. K. Kitanidis, and P. V. Roberts. Analysis of one-dimensional solute transport through porous media with spatially variable retardation factor. Water Resources Research, 26(3):437-446, 1990.

[18] S.P. Chu and H. Elliott. Latitude versus depth simulations of ecodynamics and dissolved gas chemistry relationships in the central pacific. Journal of Atmospheric Chemistry, 40(3):305-333, 2001.

[19] D.M. Cooper, A. Jenkins, R. Skeffington, and B. Gannon. Catchmentscale simulation of stream water chemistry by spatial mixing: Theory and application. Journal of Hydrology, 233(1-4):4121-137, 2000.

[20] B. B. Dykaar and P. K. Kitanidis. Macrotransport of a biologically reacting solute through porous media. Water Resources Research, 32(2): 307-320, 1996. 
[21] P. Engesgaard and K. L. Kipp. A geochemical transport model for redoxcontrolled movement of mineral fronts in groundwater flow systems - A case of nitrate removal by oxidation of pyrite. Water Resources Research, 28(10):2829-2843, 1992.

[22] P. Engesgaard and R. Traberg. Contaminant transport at a waste residue deposit. 2. Geochemical transport modeling. Water Resources Research, 32(4):939-951, 1996.

[23] T. C. Harmon, W. P. Ball, and P. V. Roberts. Nonequilibrium transport of organic contaminants in groundwater. In B. L. Sawhney and K. Brown, editors, Reactions and Movement of Organic Chemicals in Soils, pages 405-438. Soil Science Society of America, Inc., Madison, Wisconsin, 1989.

[24] J. Herzer and W. Kinzelbach. Coupling of transport and chemical processes in numerical transport models. Geoderma, 44:115-127, 1989.

[25] K.S. Hunter, Y.F. Wang, and P.M. Van Cappellen. Kinetic modeling of microbially-driven redox chemistry of subsurface environments: Coupling transport, microbial metabolism and geochemistry. Journal of Hydrology, 209(1-4):53-80, 1998.

[26] M.Z. Jacobson. Fundamentals of Atmospheric Modeling. Cambridge University Press, 1998.

[27] A. A. Jennings, D. J. Kirkner, and T. L. Theis. Multicomponent equilibrium chemistry in groundwater quality models. Water Resources Research, 18(4):1089-1096, 1982.

[28] J. J. Kaluarachchi and J. Morshed. Critical assessment of the operator splitting technique in solving the advection dispersion reaction equation. 1. First-order reaction. Advances in Water Resources, 18(2):89-100, 1995.

[29] J. F. Kanney, C. T. Miller, and D. A. Barry. Comparison of fully coupled approaches for approximating nonlinear transport and reaction problems. Advances in Water Resources, (In Press), 2002.

[30] J. Kim and S. Y. Cho. Computation accuracy and efficiency of the timesplitting method in solving atmospheric transport-chemistry equations. Atmospheric Environment, 31(15):2215-2224, 1997.

[31] A.A. Koelmans, A. Van der Heijde, L.M Knijff, and R.H. Aalderink. Integrated modelling of eutrophication and organic contaminant fate \& effects in aquatic ecosystems. A review. Water Research, 35(15):3517-3536, 2001.

[32] R. Kopmann and Markofsky M. Three-dimensional water quality modelling with TELEMAC-3D. Hydrological Processes, 14(13):2279-2292, 2000.

[33] A.S. Koziol and J.A. Pudykiewicz. Global-scale environmental transport of persistent organic pollutants. Chemosphere, 45(8):1181-1200, 2001.

[34] S. Krishnaswamy, R.D. Gunn, and P.K. Agarwal. Low-temperature oxidation of coal, 2. An experimental and modelling investigation using a fixed-bed isothermal flow reactor. Fuel, 75(3):344-352, 1996.

[35] L. W. Lake. Enhanced Oil Recovery. Prentice Hall, Englewood Cliffs, NJ, 1989. 
[36] D. Lanser and Verwer J.G. Analysis of operator splitting for advectiondiffusion-reaction problems from air pollution modelling. Journal of Computational and Applied Mathematics, 111(1-2):201-216, 1999.

[37] R. J. LeVeque and J. Oliger. Numerical methods based on additive splittings for hyperbolic partial differential equations. Mathematics of Computation, 40(162):469-497, 1983.

[38] D. M. Linn. Sorption and Degradation of Pesticides and Organic Chemicals in Soil. Soil Science Society of America, Inc., and American Society of Agronomy, Inc., Madison, WI, 1993.

[39] C. W. Liu and T. N. Narasimhan. Redox-controlled multiple-species reactive chemical transport 1. Model development. Water Resources Research, 25(5):869-882, 1989.

[40] K. T. MacQuarrie, E. A. Sudicky, and E. O. Frind. Simulation of biodegradable organic contaminants in groundwater, 1 . Numerical formulation in principal directions. Water Resources Research, 26(2):207-222, 1990.

[41] A. Mahadevan and D. Archer. Modeling the impact of fronts and mesoscale circulation on the nutrient supply and biogeochemistry of the upper ocean. Journal of Geophysical Research-Oceans, 105(C1):1209$1225,2000$.

[42] W. W. Mcnab and T. N. Narasimhan. A multiple species transport model with sequential decay chain interactions in heterogeneous subsurface environments. Water Resources Research, 29(8):2737-2746, 1993.

[43] G. J. McRae, W. R. Goodin, and J. H. Seinfeld. Numerical solution of the atmospheric diffusion equation for chemically reacting flows. Journal of Computational Physics, 45:1-42, 1982.

[44] C. T. Miller, G. Christakos, P. T. Imhoff, J. F. McBride, J. A. Pedit, and J. A. Trangenstein. Multiphase flow and transport modeling in heterogeneous porous media: Challenges and approaches. Advances in Water Resources, 21(2):77-120, 1998.

[45] C. T. Miller and A. J. Rabideau. Development of split-operator, PetrovGalerkin methods to simulate transport and diffusion problems. Water Resources Research, 29(7):2227-2240, 1993.

[46] P. Mizsey, A. Cuellar, E. Newson, P. Hottinger, T. Truong, and F. von Roth. Fixed bed reactor modelling and experimental data for catalytic dehydrogenation in seasonal energy storage applications. Computers 83 Chemical Engineering, 23:S379-S382, 1999.

[47] J. Morshed and J. J. Kaluarachchi. Critical assessment of the operator splitting technique in solving the advection dispersion reaction equation 2. Monod kinetics and coupled transport. Advances in Water Resources, 18(2):101-110, 1995.

[48] D.J. Mossman and N. AlMulki. One-dimensional unsteady flow and unsteady pesticide transport in a reservoir. Ecological Modelling, 89(1-3): 259-267, 1996.

[49] E. S. Oran and J. P. Boris. Numerical Simulation of Reactive Flow. 
Elsevier, New York, 1987.

[50] D.L. Parkhurst. User's Guide to PHREEQC: A Computer Program for Speciation, Reaction-Path, Advective-Transport and Diffusion Problems. U.S. Geological Survey, 1995. Water Resource Investigation Report 954227.

[51] J. A. Pedit. An investigation of heterogeneous sorption by a natural solid. PhD thesis, University of North Carolina, Chapel Hill, NC, 1994.

[52] G. A. Pope and M. Bavière. Basic Concepts in Enhanced Oil Recovery Processes. Elsevier, New York, 1991.

[53] H. Prommer, D. A. Barry, and G. B. Davis. A one-dimensional reactive multi-component transport model for biodegradation of petroleum hydrocarbons in groundwater. Environmental Modelling and Software, 14: 213-223, 1999.

[54] H. Prommer, G.B. Davis, and D.A. Barry. PHT3D - A three-dimensional biogeochemical transport model for modelling natural and enhanced rememdiation. In C.D. Johnston, editor, Contaminated Site Remediation: Challenges Posed by Urban and Industrial Contaminants, pages 351-358, Fremantle, WA, 21-25 March 1999.

[55] F. Rocha and A. Walker. Simulation of the persistence of atrazine in soil at different sites in portugal. Weed Research, 35:179-186, 1995.

[56] J. Rubin. Transport of reacting solutes in porous media: Relation between mathematical nature of problem formulation and chemical nature of reactions. Water Resources Research, 19(5):1231-1252, 1983.

[57] R.L. Runkel, K.E. Bencala, R.E. Broshears, and S.C. Chapra. Reactive solute transport in streams. 1. Development of an equilibrium-based model. Water Resources Research, 32(2):409-418, 1996.

[58] M.W. Saaltink, J. Carrera, and C. Ayora. On the behavior of approaches to simulate reactive transport. Journal of Contaminant Hydrology, 48: 213-235, 2001.

[59] D. Schafer, W. Schafer, and W. Kinzelbach. Simulation of reactive processes related to biodegradation in aquifers. 1. Structure of the threedimensional reactive transport model. Journal of Contaminant Hydrology, 31(1-2):167-186, 1998.

[60] T.D. Scheibe, Y.J. Chien, and J.S. Radtke. Use of quantitative models to design microbial transport experiments in a sandy aquifer. Ground Water, 39(2):210-222, 2001.

[61] L. F. Shampine and M. W. Reichelt. The MATLAB ODE suite. SIAM Journal of Scientific Computing, 18(1):1-22, 1997.

[62] J. Simunek and D. L. Suarez. Two-dimensional transport model for variably saturated porous media with major ion chemistry. Water Resources Research, 30(4):1115-1133, 1994.

[63] H.B. Singh. Composition, Chemistry, and Climate of the Atmosphere. John Wiley and Sons, New York, 1995.

[64] B. Sportisse, G. Bencteux, and P. Plion. Method of lines versus operator splitting for reaction-diffusion systems with fast chemistry. Environmen- 
tal Modelling and Software, 15(6-7):673-679, 2000.

[65] C. I. Steefel and K. T. B. MacQuarrie. Approaches to modeling of reactive transport in porous media. In P. C. Lichtner, C. I. Steefel, and E. H. Oelkers, editors, Reactive Transport in Porous Media: General Principles and Applications to Geochemical Processes, pages 83-129. Mineralogical Society of America, Washington, DC, 1996.

[66] C. I. Steefel and S.B. Yabusaki. OS3D/GIMRT, Software for Modelling Multicomponent-Multidimensional Reactive Transport, User Manual and Programmer's Guide. Pacific Northwest Laboratory, Richland, Washington, 1995.

[67] D.L. Stefanovic and H.G. Stefan. Accurate two-dimensional simulation of advective-diffusive-reactive transport. Journal of Hydraulic EngineeringASCE, 127(9):728-737, 2001.

[68] G. Strang. On the construction and comparison of difference schemes. SIAM Journal on Numerical Analysis, 5(3):506-517, 1968.

[69] N.-Z. Sun. Mathematical Modeling of Groundwater Pollution. SpringerVerlag, New York, 1996.

[70] A. J. Valocchi and M. Malmstead. Accuracy of operator splitting for advection-dispersion-reaction problems. Water Resources Research, 28 (5):1471-1476, 1992.

[71] A. L. Walter, E. O. Frind, D. W. Blowes, C. J. Ptacek, and J. W. Molson. Modeling of multicomponent reactive transport in groundwater 1 . Model development and evaluation. Water Resources Research, 30(11):31373148, 1994.

[72] K.Y. Wang, J.A. Pyle, and D.E. Shallcross. Formulation and evaluation of ims, an interactive three-dimensional tropospheric chemical transport model 1. Model emission schemes and transport processes. Journal of Atmospheric Chemistry, 38(2):195-227, 2001.

[73] M. F. Wheeler and C. N. Dawson. An operator-splitting method for advection-diffusion-reaction problems. Technical Report Technical Report 87-9, Rice University Department of Computational and Applied Mathematics, Houston, TX, 1987.

[74] M. F. Wheeler, C. N. Dawson, P. B. Bedient, C. Y. Chiang, R. C. Borden, and H. S. Rifai. Numerical simulation of microbial biodegradation of hydrocarbons in ground water. In Proceedings of the NWWA Conference on Solving Ground Water Problems with Models, pages 92-109, 1987.

[75] T. M. Wood and A. M. Baptista. A model for diagnostic analysis of estuarine geochemistry. Water Resources Research, 29(1):51-71, 1993.

[76] T. Xu, J. Samper, C. Ayora, M. Manzano, and E. Custidio. Modeling of non-isothermal multi-component reactive transport in field scale porous media flow systems. Journal of Hydrology, 214(1-4):144-164, 1999.

[77] G. T. Yeh and V. S. Tripathi. A critical evaluation of recent developments in hydrogeochemical transport models of reactive multichemical components. Water Resources Research, 25(1):93-108, 1989.

[78] G.-T. Yeh and V. S. Tripathi. A model for simulating transport of reactive 
multispecies components: Model development and demonstration. Water Resources Research, 27(12):3075-3094, 1991.

[79] A. Zysset and F. Stauffer. Modeling of microbial processes in groundwater infiltration systems. In T. F. Russell, R. E. Ewing, C. A. Brebbia, W. G. Gray, and G. F. Pinder, editors, Mathematical Modeling in Water Resources, volume 2, pages 325-332, Southampton, UK, 1992. Computational Mechanics Publications.

[80] A. Zysset, F. Stauffer, and T. Dracos. Modeling of chemically reactive groundwater transport. Water Resources Research, 30(7):2217-2228, 1994. 